East African Medical Journal Vol. 85 No. 10 October 2008

UTILITY OF LIVER BIOPSY IN HIV-INFECTED PATIENTS WITH FEBRILE ILLNESSES AND INCONCLUSIVE EVALUATION

J. Shavadia, MBChB, Resident, S. Mwanzi, MBChB, Resident, F. Rana, MBChB, MMed (Path), Associate Professor, Department of Anatomic Pathology, M. Twahir, MBChB, MMed, Cert. Nephrology, Consultant Physician and Nephrologist, The Aga Khan University Hospital Nairobi, P. O. Box 40374-00100, Nairobi, Kenya

Request for reprints to: Dr. J. Shavadia, The Aga Khan University Hospital Nairobi, P. O. Box 40374-00100, Nairobi, Kenya

\title{
UTILITY OF LIVER BIOPSY IN HIV-INFECTED PATIENTS PRESENTING WITH FEBRILE ILLNESSES AND INCONCLUSIVE EVALUATION
}

\author{
J. SHAVADIA, S. MWANZI, F. RANA and M. TWAHIR
}

\begin{abstract}
Objectives: To determine the utility of liver biopsy in providing a diagnosis in HIVinfected patients presenting with febrile illnesses and inconclusive initial investigative work up.

Design: A retrospective descriptive study.

Setting: The Aga Khan University Hospital, Nairobi.

Subjects: Twelve in-patients with HIV disease who underwent liver biopsy following inconclusive initial investigative work up for febrile illnesses between January and December 2007.

Results: Seven out of 12 patients had granulomatous hepatitis reported on histology with characteristic tuberculous epitheloid granulomas all having stainable acid-alcohol fast bacilli on Ziehl-Nielsen (ZN) stain. The mean alkaline phosphatase (ALP) and gamma glutamyl transpeptidase (GGT) levels in these seven patients were 260U/L and $304 \mathrm{U} / \mathrm{L}$ respectively, while the mean aspartate aminotransferase (SGOT) and alanine aminotransferase (SGPT) were 106U/L and 72U/L respectively.

Conclusion: Disseminated tuberculosis is still among the most common causes of unexplained pyrexia in our HIV- infected cohort and a liver biopsy, performed earlier in the investigative work up of unexplained fever in the HIV-infected patient, would be a useful adjunct in providing a diagnosis.
\end{abstract}

\section{INTRODUCTION}

Physicians taking care of patients with Human Immunodeficiency Virus (HIV) infection routinely come across patients presenting with febrile illnesses and are posed not only with the challenge of making an appropriate diagnosis but initiating evidence-based therapy especially when the initial 'septic-screen' is reported normal.

With differential diagnosis ranging from infections to malignancies to less common connective tissue disorders among others, most patients undergo first line investigations including a full blood count, malaria slides, sputum microscopy and culture, urinalysis, chest radiographs and blood cultures since the commoner cause of a febrile illness in HIV infected patients in our settings is infective. If these non-invasive investigations are non conclusive, patients are then subjected to lymph node biopsies (if present and accessible), bone marrow aspirate (BMA) and cultures and a liver biopsy.

In clinical practice, due to the endemicity of tuberculosis in the HIV infected population, the atypically bland manner of its presentation, and the significant morbidity, mortality and public health concern associated with untreated tuberculosis, most patients would be initiated on an empirical 'trial of anti- tuberculous therapy' if the initial septic screen is reported normal.

Autopsy studies done in Kenya on HIV infected adults found previously undiagnosed tuberculosis present in $50 \%$ of the cases, $80 \%$ of whom had disseminated disease. In over half of the patients who had disseminated disease, tuberculous granulomas were evident in the liver (1).

We therefore decided to evaluate the role of a liver biopsy in HIV infected patients presenting with a febrileillness and having an inconclusive diagnostic work up. 


\section{MATERIALS AND METHODS}

Aretrospectivestudy performed between January and December 2007 at The Aga Khan University Hospital, Nairobi, where twelve HIV infected patients who presented with a febrile illness for a mean duration of 2.1 weeks, underwent the following investigations as part of the initial septic screen:

(i) Full blood count and malaria slides.

(ii) Sputum (induced with 3\% saline, if cough was non productive) for microscopy and a Ziehl Nielsen stain.

(iii) Urine and blood cultures obtained prior to initiation of empirical antibiotics.

(iv) Chest radiograph.
Patients, who had inconclusive initial septic screen, underwent a repeat physical examination looking particularly for evidence of lymphadenopathy. Patients who did not have enlarged lymph nodes or declined consent for excision underwent a bone marrow aspirate (BMA) followed by a diagnosticliver biopsy if the BMA cytology was inconclusive.

Baseline characteristics: The median age of the cohort was 40 years (range 32-47years) and sex ratio distributed as $\mathrm{M}: \mathrm{F}=1: 2$. The baseline characteristics of all twelve patients are shown in Table 1 . The pertinent liver function tests are illustrated in Table 2.

Table 1

Baseline characteristics

\begin{tabular}{lcccccccc}
\hline Age & Sex & WBC & LYM & ESR & CXR & URINE C/S & BLOOD C/S & CD4 \\
\hline 41 & M & 6.5 & $6 \%$ & 50 & N & NG & NG & 42 \\
40 & $\mathrm{~F}$ & 6.4 & $13 \%$ & 72 & N & NG & NG & NA \\
43 & M & 6.7 & $22 \%$ & 43 & NON SP INF & NG & NG & 215 \\
33 & $\mathrm{~F}$ & 5.9 & $43 \%$ & 38 & N & NG & NA & NA \\
43 & M & 2.9 & $41 \%$ & 47 & N & NG & NG & 28 \\
40 & $\mathrm{~F}$ & 2.2 & $31 \%$ & 15 & N & NG & NG & 13 \\
39 & $\mathrm{~F}$ & 4.2 & $54 \%$ & 36 & $\mathrm{~N}$ & $\mathrm{NG}$ & $\mathrm{NG}$ & $\mathrm{NA}$ \\
47 & $\mathrm{M}$ & 5.9 & $9 \%$ & 30 & $\mathrm{~N}$ & $\mathrm{NG}$ & $\mathrm{NG}$ & 490 \\
35 & $\mathrm{~F}$ & 7.2 & $4 \%$ & 70 & $\mathrm{~N}$ & $\mathrm{NG}$ & $\mathrm{NG}$ & 50 \\
30 & $\mathrm{~F}$ & 5.7 & $10 \%$ & 64 & $\mathrm{~N}$ & $\mathrm{NG}$ & $\mathrm{NG}$ & 13 \\
32 & $\mathrm{~F}$ & 1.6 & $9 \%$ & 68 & $\mathrm{~N}$ & $\mathrm{NG}$ & $\mathrm{NG}$ & 314 \\
42 & $\mathrm{~F}$ & 6.3 & $20 \%$ & 65 & $\mathrm{~N}$ & $\mathrm{NG}$ & $\mathrm{NG}$ & 73 \\
\hline
\end{tabular}

Age $=$ Expressed in years

Sex $=\mathrm{M}$ - male $\mathrm{F}-$ female

WBC $=$ White cell count

LYM = Lymphocyte

$\mathrm{CXR}=$ Chest X-ray

$\mathrm{NA}=$ Not available
URINE $\mathrm{C} / \mathrm{S}=$ Urine culture and sensitivity

BLOOD $\mathrm{C} / \mathrm{S}=$ Blood culture and sensitivity

CD4 = CD4 count (cells $/ \mathrm{mm}^{3}$ )

ESR $=$ Erythrocyte sedimentation rate

NON SP INF $=$ Non specific infective process

NG $\quad$ No growth

Table 2

Liver function tests

\begin{tabular}{lccc}
\hline ALP & GGT & SGOT & SGPT \\
\hline 99 & 124 & 107 & 58 \\
202 & 131 & 63 & 47 \\
238 & 696 & 121 & 108 \\
814 & 371 & 56 & 64 \\
182 & 148 & 40 & 40 \\
192 & 471 & 63 & 41 \\
52 & 29 & 33 & 10 \\
68 & 351 & 93 & 53 \\
221 & 345 & 307 & 226 \\
216 & 417 & 80 & 62 \\
98 & 86 & 40 & 18 \\
151 & 352 & 45 & 70 \\
\hline ALP & $=$ Alkaline phosphatase (U/L) & GGT & $=$ Gamma glutamyl transpeptidase (U/L) \\
SGOT $=$ Aspartate aminotransferase (U/L) & SGPT & $=$ Alanine aminotransferase (U/L)
\end{tabular}




\section{RESULTS}

Majority of the patients in the study $(83 \%)$ had an absolute lymphopenia $\left(<1.5 \times 10^{9} / \mathrm{L}\right)$ with a mean lymphocyte count of $1.01 \times 10^{9} / \mathrm{L}\left(0.14-2.54 \times 10^{9} / \mathrm{L}\right)$. This was reflected in the mean $\mathrm{CD}_{4}$ count of 137 cells / $\mathrm{mm}^{3}\left(13-490\right.$ cell $\left./ \mathrm{mm}^{3}\right)$. The mean ESR for our study patients was $50 \mathrm{~mm} /$ hour $(15-72 \mathrm{~mm} /$ hour $)$.

All patients, who had blood and urine cultures drawn prior to initiation of empirical antibiotic therapy, had no growth at 48 hours. All chest radiographs were reported as normal, except one who had a non specific infective pneumonitis. The mean alkaline phopshatase (ALP) and gamma glutamyl transpeptidase (GGT) of the cohort was 211U / Land 293U / L respectively. Liver transaminases were elevated in $75 \%$ of the patients with the mean aspartate aminotransferase (SGOT) at 67U/L and alanine aminotransferase (SGPT) at 64U/L.

All but one patient had either no evidence or non- accessible lymphadenopathy. The one patient had anterior cervicallymphadenopathy but declined consent for an excisional lymph node biopsy. On evaluation of the bone marrow aspirates, no patient had cytological evidence of lymphoma. One patient had M. tuberculosis while another had mycobacteria other than tuberculosis (MOTT) grown on bone marrow culture at five weeks.

All twelve patients underwent a liver biopsy since the BMA was inconclusive while bone marrow culture reports were awaited. Seven out of the 12 biopsies showed features of tuberculous hepatitis with preservation of the liver architecture. Five of the seven showed well defined classical necrotising granulomas with epithelioid cells and the remaining two showed ill defined non epithelioid focal areas of necrosis with neutrophilic infiltrate predominantly. All seven biopsies had acid alcohol fast bacilli on $\mathrm{ZN}$ staining, five on the initial sections and two on deeper sections.

Of the five cases that did not show tuberculous hepatitis, two showed steatohepatitis (one having cirrhosis), while the rest showed chronic hepatitis of mild activity and no fibrosis on the Ishaak scoring system (1).

On sub-analysis of the seven patients who had granulomas on liver biopsy, the mean ALP and GGT levels were $260 \mathrm{U} / \mathrm{L}$ and $304 \mathrm{U} / \mathrm{L}$ respectively, while the mean SGOT and SGPT were $106 \mathrm{U} / \mathrm{L}$ and $72 \mathrm{U} / \mathrm{L}$ respectively.

Ten patients were both hepatitis B surface antigen (HBsAg) and hepatitis C antibody (anti $\mathrm{HCV}$ antibody) negative. The HBsAg and anti HCV antibody results of the remaining two patients were not available. However, all patients who had granulomatous hepatitis were $\mathrm{HBsAg}$ and anti $\mathrm{HCV}$ negative.

\section{DISCUSSION}

Fever of unknown or uncertain origin (FUO) constitutes a common clinical challenge in patients infected with HIV. It becomes more frustrating after the initial investigations fail to identify the source of their fever, as this not only increases patient hospital stay but also puts them through more invasive investigative work-up. Our study results were similar to results from Rana et al (2) who reported from the samegeographical setting, and in close approximation to results from Nigeria where $65 \%$ of HIV infected patients who underwent a post-mortem liver biopsy had evidence of tuberculosis (3).

García-Ordóñez et al (4) found tuberculosis in $50 \%$ of their percutaneous liver biopsies from HIVinfected patients presenting with FUO. They also reported leishmaniasis in $20 \%$ of their cohort. Despite the existence of leishmaniasis endemic areas and publications of leishmania outbreaks in Kenya (5-7), we interestingly did not find any case of leishmaniasis in our HIV-infected cohort.

Other studies in developing countries have however reported a much lower prevalence of tuberculous hepatitis in HIV infected patients. Amarapurkar et al (8) demonstrated a prevelance rate of about $36 \%$ while Piratvisuth et al (9) had a prevalence rate of $33 \%$.

From our study cohort, patients who had tuberculous hepatitis on biopsy had a two-fold increase in their mean liver transaminase levels, and a several fold increase in their ALP and GGT levels. The results of this study, although limited by its small numbers, demonstrate that a liver biopsy would increase the diagnostic yield for mycobacterial infections in patients with HIV / AIDS presenting with a febrile illness and a non diagnostic initial work up. This is especially so in patients who also have derangements in their liver function tests. All patients who had granulomatous hepatitis were treated for $\mathrm{M}$. tuberculosis, except one patient whose bone marrow culture was positive for MOTT.

We therefore recommend that a liver biopsy be performed earlier in the diagnostic hierarchy of unexplained fever in HIV - infected patients, since it leads to a change in the clinical management of such patients.

\section{REFERENCES}

1. Ishak, K., Baptista, A., Bianchi, L., et al. Histological grading and staging of chronic hepatitis. J. Hepatol. 1995; 22: 696-699.

2 Rana, F.S., Hawken, M.P., Mwachari, C., et al. Autopsy study of HIV-1-positive and HIV-1-negative adult medical patients in Nairobi, Kenya. J. Acquir. Immune Defic. Syndr. 2000; 24: 23-29. 
3 Echejoh, G.O., Mandong, B.M., Tanko, M. N., et al. Hepatic histopathological findings in HIV patients at postmortem in Jos University Teaching Hospital, Nigeria. Trop. Doct. 2006; 36: 228-231.

4 García-Ordóñez, M. A. Colmenero, J. D. JiménezOñate., F. et al. Diagnostic usefulness of percutaneous liver biopsy in HIV-infected patients with fever of unknown origin. J. Infect. 1999; 38: 94-98.

5 Jahn, A., Lelmett, J.M. and Diesfeld, H.J Seroepidemiological study on kala-azar in Baringo District, Kenya. J. Trop. Med. Hyg. 1986; 89: 91-104.

6 Ngoka, J.M. and Mutinga, M.J. Visceral leishmaniasis in Kenya: the onset of an epidemic outbreak in the
Machakos District of Kenya. East Afr. Med. J. 1978; 55: 328-331.

7 Boussery, G., Boelaert, M., van Peteghem, J., et al Visceral leishmaniasis (kala-azar) outbreak in Somali refugees and Kenyan shepherds, Kenya. Emerg. Infect. Dis. 2001; 7(Suppl) 3: 603-604 .

8 Amarapurkar, A.D. and Sangle, N.A. Histological spectrum of liver in HIV-autopsy study. Ann. Hepatol. 2005; 4: 47-51.

9 Piratvisuth, T., Siripaitoon, P., Sriplug, H., et al. Findings and benefit of liver biopsies in 46 patients infected with human immunodeficiency virus. J. Gastroenterol. Hepatol. 1999; 14:146-149. 